\title{
Intoxicação cúprica acumulativa em búfalos
}

\section{Accumulative copper poisoning in buffaloes}

\author{
Antonio Humberto Hamad Minervino항 Raimundo Alves Barrêto Júnior ${ }^{1}$; Rodrigo \\ Nogueira Fernandes Ferreira; João Paulo Elsen Saut ${ }^{1}$; Enoch Brandão de Sousa \\ Meira Júnior'; Frederico Augusto Mazzocca Lopes Rodrigues'; Tizianne Larissa \\ Duim Ribeiro Nakagawa ${ }^{2}$; Selwyn Arlington Headley ${ }^{3}$; Enrico Lippi Ortolani ${ }^{1 *}$
}

\section{Resumo}

O presente trabalho objetivou analisar o quadro clínico, variáveis metabólicas e a concentração de cobre nos tecidos de bufalinos submetidos à intoxicação cúprica acumulativa (ICA). Foram utilizados 10 bufalinos jovens, da raça Murrah, aleatoriamente distribuídos em seis animais no grupo suplementado com cobre (BUFCu) e quatro no grupo controle (BUF). O grupo BUFCu recebeu suplementação progressiva de cobre através de administração diária de solução aquosa deste microelemento, por meio da fistula ruminal. A dose diária inicial era de $2 \mathrm{mg} \mathrm{de} \mathrm{Cu} / \mathrm{kgPV}$ (CuSO4.5H2O), durante 7 dias, sendo esta dose de cobre aumentada em $2 \mathrm{mg} / \mathrm{kgPV}$ a cada semana, até o término do experimento (105 dias). Foram realizadas três biópsias hepáticas (dias 0 - 45-105) nos animais para determinação da concentração de $\mathrm{Cu}$ e de $\mathrm{Zn}$. Quinzenalmente, foi realizado exame clínico, pesagem e coleta de sangue. Dois búfalos do grupo BUFCu manifestaram quadro clínico fatal sugestivo de ICA. Destaca-se a presença de dois quadros clínicos diferentes, o clássico $(n=1)$ e um atípico $(n=1)$, caracterizado pelo acúmulo destacado de cobre hepático, hiporexia progressiva seguida de anorexia, desidratação, oligúria, apatia acentuada e morte, mas sem apresentar hemoglobinúria. Quatro búfalos foram resistentes à ICA a despeito da administração de altas quantidades de cobre. Em búfalos que apresentem quadros clínicos similares ao quadro descrito como atípico, a ICA pode ser considerada como um possível diagnóstico mesmo sem a observação macroscópica de hemoglobinúria.

Palavras-chave: Cobre, sintomas, fígado, búfalo

\begin{abstract}
The main objective of this study was to evaluate the clinical and hematological alterations, and the hepatic concentration of $\mathrm{Cu}$ in buffaloes with chronic copper poisoning (CCP). Ten buffalo yearling steers were randomly distributed into two groups: one copper supplemented (BUFCu; $n=6)$ and another control group (BUF; $\mathrm{n}=4$ ). The group BUFCu received, by ruminal fistula, $2 \mathrm{mg} \mathrm{Cu} / \mathrm{kgBW}$ (as $\mathrm{CuSO} 4.5 \mathrm{H} 2 \mathrm{O}$ ) daily during one week; after which $2 \mathrm{mg} \mathrm{Cu} / \mathrm{kgBW}$ was added during each additional week, until the end of the experiment $\left(105^{\text {th }}\right.$ day). Three liver biopsies were realized during the experiment (day zero, $45^{\text {th }}$, and $105^{\text {th }}$ day) to determine the degree of copper accumulation. Alterations in body weight, clinical examination, and hematological values were monitored every 15 days. Two buffaloes supplemented with copper demonstrated clinical manifestations consistent with CCP, and died. Two distinct clinical
\end{abstract}

\footnotetext{
1 Departamento de Clínica Médica. Faculdade de Medicina Veterinária e Zootecnia - FMVZ. Universidade de São Paulo - USP. Av. Prof. Orlando Marques de Paiva, 87, Cidade Universitária, 05508-900, São Paulo, SP, Brasil. * Autor para correspondência, e-mail: ortolani@usp.br

2 Med. Veterinária Residente na área de Patologia Animal da Universidade Estadual de Londrina.

3 Department of Basic Veterinary Sciences, School of Veterinary Medicine, St. Matthew's University, Grand Cayman, Cayman Islands, BWI.

* Autor para correspondência
} 
manifestations were observed, one classical $(\mathrm{n}=1)$ and another atypical $(\mathrm{n}=1)$, characterized by remarkable high levels of liver copper, progressive hyporexia followed by anorexia, dehydration, severe apathy, decreased rumen movements, oliguria, and death. Some animals were resistant to CCP although high copper intake. In buffalos with clinical picture similar to the one described as atypical, CCP should be considered as a possible diagnostic even without presence of macroscopic hemoglobinuria.

Key words: Copper, clinical manifestations. liver, buffaloes

\section{Introdução}

O Cobre $(\mathrm{Cu})$, quando ingerido em concentrações acima do requerimento e da capacidade de excreção pelo organismo, se acumula progressivamente no fígado sem causar nenhum sintoma de intoxicação, até atingir um limiar máximo, quando é abruptamente liberado na corrente circulatória como cobre livre, que irá provocar degeneração hepática, hemólise intensa, icterícia e morte, sendo esta enfermidade denominada de intoxicação cúprica acumulativa (ICA) (MACHADO, 1998; ORTOLANI, 2003; SOARES, 2004).

Em um estudo retrospectivo em laboratório de investigação toxicológica durante cinco anos, com várias espécies, observou-se elevado número de amostras com suspeita de intoxicação por cobre, sendo este diagnóstico confirmado em boa parte destas, onde o cobre destacou-se como um dos elementos minerais mais importantes envolvidos em casos de intoxicação juntamente com o chumbo (HOOF; BOERMANS; BAIRD, 1998). Alerta-se para o fato de que a intoxicação por cobre pode estar ocorrendo em um número muito maior de casos, visto que o diagnóstico confirmativo nem sempre é estabelecido ou que o mesmo seja confundido com outras enfermidades que causem quadros hemolíticos (BIDEWELL; LIVESEY, 2002).

Entre os casos de intoxicação por cobre descritos na literatura, certa variabilidade de sintomas foi descrita. De uma maneira geral, os ruminantes apresentam hiporexia, anorexia, perda de peso, desidratação, icterícia, hemoglobinúria e morte (AUZA et al., 1999; ZICARELLI et al., 1981).

A ICA é classicamente conhecida como uma enfermidade de grande importância na espécie ovina (ORTOLANI, 2003) e vem despontado como doença emergente em bovinos (BIDEWELL; LIVESEY, 2002). Em bufalinos, apenas um caso relata o acometimento por esta enfermidade (ZICARELLI et al., 1981), onde bezerros búfalos que recebiam sucedâneo lácteo rico em cobre demonstraram após alguns meses sinais de ICA, seguidos de morte, onde os autores sugerem que os búfalos são mais susceptíveis à ICA do que os bovinos. Cardoso et al. (1997) sugerem que búfalos possuem uma maior absorção de cobre do que bovinos, o que indica também uma maior susceptibilidade, entretanto este estudo foi conduzido com níveis baixos ou normais de cobre na dieta, sendo que não existem informações sobre búfalos recebendo altas doses de cobre.

No estado de São Paulo, em especial, onde grande parte das criações de búfalos é voltada para a produção de leite (MACEDO et al., 2001), o risco de ICA é ainda maior, pois os animais são submetidos a condições mais intensivas de criação. Assim, com o surgimento recente de diversos casos de ICA em bovinos, estranha-se o fato de não haver relatos em búfalos.

Deste modo, o presente estudo objetivou avaliar a presença de quadro clínico de ICA, os sintomas manifestados e as alterações em variáveis clínicas e histopatológicas de búfalos submetidos a doses crescentes de cobre.

\section{Material e Métodos}

O presente estudo foi aprovado pela comissão de bioética da Faculdade de Medicina Veterinária e Zootecnia da Universidade de São Paulo, em 25 de outubro de 2005 (Protocolo número 746/2005).

Foram utilizados 10 búfalos hígidos da raça Murrah entre 8 e 10 meses de idade e pesando entre 
150 e $180 \mathrm{~kg}$ no início do experimento. Dois meses antes do início do período experimental, todos os animais foram vermifugados, receberam banho com carrapaticida e foram vacinados contra tétano e clostridioses, bem como submetidos à cirurgia para colocação de cânula ruminal de látex.

Os animais foram mantidos em baias individuais em sistema de "tie-stall", nas dependências do Departamento de Clínica Médica da Faculdade de Medicina Veterinária e Zootecnia -FMVZ da Universidade de São Paulo - USP, Campus de São Paulo e receberam ração total composta de $75 \%$ da matéria seca (MS) de feno de capim Coastcross e $25 \%$ da MS de ração concentrada (14\% de PB), calculada em relação a $2,7 \%$ do peso vivo individual. Os búfalos tiveram livre acesso à água e diariamente receberam $35 \mathrm{~g}$ de sal mineralizado comercial, adicionado à ração total.

O experimento foi inteiramente ao acaso onde os búfalos foram sorteados em dois grupos compostos de quatro animais no grupo controle (BUF) e seis no grupo suplementado com solução de sulfato de cobre (BUFCu).

O modelo proposto para estudar o acúmulo de cobre no organismo foi adaptado ao descrito por Machado (1998) para ovinos, onde os animais do grupo $\mathrm{BUFCu}$ receberam diariamente solução de sulfato de cobre pentahidratado $\left(\mathrm{CuSO}_{4} 5 \mathrm{H}_{2} \mathrm{O}\right)$, administrada diretamente no rúmen através da cânula ruminal. A dose inicial utilizada foi de $2 \mathrm{mg}$ de $\mathrm{Cu} / \mathrm{kgPV}$, sendo esta acrescida de mais $2 \mathrm{mg} / \mathrm{kg} /$ PV a cada semana até o final do experimento, após 7 quinzenas. Nos casos de intoxicação cúprica a administração de sulfato de cobre foi imediatamente interrompida, com subseqüente tratamento com 3,4 $\mathrm{mg} / \mathrm{kg}$ de peso vivo de tetratiomolibdato de amônia (TTM), segundo preconizam Ortolani (2003) e Soares (2004).

Foram realizadas três séries de biópsias hepáticas em todos os animais em experimentação para determinação da concentração de cobre neste órgão-estoque. A $1^{\mathrm{a}}$ coleta foi realizada no início do experimento, antes do oferecimento da primeira dose de cobre, a $2^{\mathrm{a}}$ coleta após 45 dias e a $3^{\mathrm{a}}$ coleta após 105 dias (7 quinzenas), no final do experimento. Nos animais que sucumbiram, a despeito do tratamento, foram coletadas amostras de fígado do lobo cranial, imediatamente após a morte. As amostras hepáticas foram secas e digeridas e as concentrações de cobre e zinco foram determinadas através de espectrofotometria de absorção atômica, segundo Ortolani (1997). Amostras de fígado de todos os animais, nas diferentes coletas, foram fixadas em solução de formalina a $10 \%$ e processadas para histopatologia de rotina.

Os animais eram constantemente observados no momento da infusão da solução com cobre na fistula ruminal e semanalmente era realizado exame clínico dos mesmos. Quando os animais apresentavam alguma alteração de comportamento, em especial uma redução do consumo de alimentos, este exame clínico passava a ser mais freqüente, sendo realizado diariamente exame macroscópico da urina para verificar se os animais apresentavam hemoglobinúria.

Foram realizadas pesagens e coletas de sangue quinzenalmente em todos os animais até o final do período experimental, totalizando 8 coletas por animal. A primeira delas foi feita antes dos animais receberem a $1^{\mathrm{a}}$ dose de cobre e as demais a cada uma das sete quinzenas de experimento. As amostras de sangue foram obtidas por meio de venipunctura jugular externa, utilizando-se tubos de coleta a vácuo. Para as determinações sangüíneas do cobre, aspartato aminotransferase (AST), gama glutamiltransferase (GGT), uréia e creatinina, o sangue foi coletado em tubos sem anticoagulante para a obtenção do soro, que foi armazenado em freezer à $20{ }^{\circ} \mathrm{C}$ negativos posteriormente descongelado uma única vez para realização das respectivas análises. Para a determinação do volume globular, foi coletado sangue em tubos com EDTA, mantidos em refrigeração até a análise em contador automático de células $(\mathrm{ABC} \AA$ - Animal Blood Counter) da marca $\mathrm{ABX}{ }^{\circledR}$, que ocorreu em 
no máximo 4 horas após a coleta. Os resultados das atividades das enzimas foram determinados em 30 ${ }^{\circ} \mathrm{C}$, segundo recomendação de Kaneko, Harvey e Bruss (1997).

Todos os dados foram testados quanto a sua distribuição, utilizando-se para tal o teste de Kolmogorov-Smirnov. Os dados que tiveram distribuição normal foram submetidos à análise de variância (teste F) e comparados pelo teste de Tukey. Os dados com distribuição não-paramétrica foram avaliados pelo teste de Kruskal-Wallis e expressos por mediana. Foi adotado neste trabalho um grau de significância mínimo de 5\%. A maioria das análises estatísticas foi processada com o auxílio de software estatístico (MINITAB, 2000).

\section{Resultados}

Nenhum búfalo do grupo controle apresentou qualquer alteração clínica durante o experimento. Entretanto, dentre os animais do grupo suplementado com cobre, dois búfalos manifestaram, no decorrer da penúltima quinzena, quadro clínico fatal sugestivo de ICA. A tabela 1 apresenta as variáveis clínicas estudadas nos dois animais intoxicados na última coleta. Um búfalo apresentou quadro clínico considerado clássico, com presença de hemoglobinúria, apatia, andar cambaleante, diminuição dos movimentos ruminais, desidratação grave, mucosas aparentes e pele intensamente amareladas, fezes pastosas e oligúria. Em um período de 14 dias, houve uma redução de peso vivo de 9,4\%. Mesmo após o início do tratamento com tetratiomolibdato de amônia, esse búfalo morreu no dia subseqüente a observação da crise hemolítica.

Tabela 1. Variáveis sangüíneas e teor de cobre hepático dos búfalos que apresentaram quadro clássico e atípico de intoxicação cúprica acumulativa.

\begin{tabular}{lcc}
\hline Variáveis & Búfalo 7 & Búfalo 9 \\
\hline Quadro clínico & Clássico & Atípico \\
\cline { 2 - 3 } Cobre hepático $(\mathrm{ppm})$ & 2053 & 2051 \\
Cobre sérico $(\mu \mathrm{Mol} / \mathrm{L})$ & 48,3 & 20,5 \\
GGT sérica $(\mathrm{U} / \mathrm{L})$ & 84 & 23 \\
AST sérica $(\mathrm{U} / \mathrm{L})$ & 4001 & 190 \\
Uréia $(\mathrm{mMol} / \mathrm{L})$ & 10 & 22 \\
Creatinina $(\mu \mathrm{Mol} / \mathrm{L})$ & 169 & 288 \\
Volume globular & 28 & 40 \\
\hline
\end{tabular}

$\mathrm{Na}$ necropsia, observou-se intensa coloração amarelada das membranas serosas da cavidade abdominal, fígado ictérico, friável e com os bordos arredondados; rins enegrecidos tanto na região cortical quanto na medular; e a mucosa abomasal congesta e com pequenas erosões. Na histopatologia observou-se grave fibroplasia, necrose centrolobular e estase biliar; degeneração gordurosa moderada e tumefação hepatocelular; e areas discretas de apoptose hepatocelular.

Constituiu uma surpresa o fato de um búfalo ter manifestados quadro clínico atípico de ICA, caracterizado por uma maior perda de peso vivo
(15\%) decorrente de um período maior de anorexia (cerca de 18 dias), desidratação intensa, redução dos movimentos ruminais, oligúria e marcante apatia. Diferentemente do animal que manifestou quadro clássico de ICA, o búfalo com quadro atípico não apresentou hemoglobinúria, mucosas amareladas, andar cambaleante e excreção de fezes pastosas. A confirmação diagnóstica deste quadro atípico baseou-se na concentração elevada de cobre hepático, similar a observada no animal com quadro clássico e superior aos demais animais que não apresentaram sintomas clínicos. 
À necropsia, detectou-se uma caquexia marcante, presença de conteúdo altamente ressecado no interior do rúmen, omaso, abomaso e, em menor grau, nos intestinos. O fígado se encontrava com os bordos levemente arredondados. As alterações histopatológicas eram discretas e caracterizadas por degeneração gordurosa e tumefacao hepatocellar com proliferação moderada das células epiteliais dos ductos biliares.

Devido à presença de animais intoxicado e resistentes dentro do grupo $\mathrm{BUFCu}$, optou-se pela subdivisão deste em dois sub-grupos: BUF ICA búfalos intoxicados e BUF R - búfalos resistentes, considerando-se apenas os valores da última quinzena.

O ganho de peso total após 105 dias foi de 48 e $12 \mathrm{~kg}$ para os animais controle e suplementados com cobre respectivamente, não sendo verificada diferença estatística devido ao elevado desvio padrão obtido. A diferença no ganho de peso só foi verificada após a divisão dos animais em sub-grupos, onde os animais resistentes e controle apresentaram ganhos superiores $(\mathrm{P}<0,04)$ aos animais que apresentaram quadros de ICA (26, $48 \mathrm{e}-21 \mathrm{~kg}$ respectivamente).
A tabela 2 apresenta os resultados de $\mathrm{Cu}$ e $\mathrm{Zn}$ no fígado dos bufalinos dos grupos controle e suplementados com cobre nas três biópsias realizadas durante o experimento. Destaca-se nesta tabela o aumento progressivo nos teores de cobre no grupo $\mathrm{BUFCu}$, enquanto que não existiu diferença dentro do grupo controle. Os ruminantes suplementados com cobre apresentaram maiores teores desse elemento que o grupo controle na $2^{\mathrm{a}}$ e na $3^{\mathrm{a}}$ coleta, após 45 e 105 dias de experimento, respectivamente. Em relação ao zinco, destaca-se a elevação da concentração hepática deste elemento na última biópsia no grupo intoxicado, em relação às duas primeiras amostragens e ao grupo controle.

A tabela 3 apresenta os resultados das concentrações de cobre e de zinco no fígado apenas na ultima biópsia e considerando os subgrupos: búfalos intoxicados (BUFCu ICA; $n=2$ ), resistentes (BUFCu R; $n=4$ ) e controle (BUF; $n=4)$. Os búfalos que apresentaram quadro de ICA tiveram maior concentração de cobre hepático $(\mathrm{P}<0,001)$ que os animais resistentes, que receberam semelhante dose de cobre durante o experimento. O mesmo não ocorreu com os teores hepáticos de zinco, havendo diferença apenas entre os grupos intoxicados e resistentes em relação ao grupo controle.

Tabela 2. Mediana da concentração hepática de cobre e zinco dos animais dos grupos suplementados com cobre $(\mathrm{BUFCu})$ e controle (BUF) nos três momentos de coleta.

\begin{tabular}{|c|c|c|c|c|c|c|}
\hline \multirow[t]{2}{*}{ Grupos } & \multicolumn{3}{|c|}{$\mathrm{Cu}(\mathrm{ppm})$} & \multicolumn{3}{|c|}{$\mathrm{Zn}(\mathrm{ppm})$} \\
\hline & zero & $45 \mathrm{~d}$ & $105 \mathrm{~d}$ & zero & $45 \mathrm{~d}$ & $105 \mathrm{~d}$ \\
\hline $\mathrm{BUFCu}$ & $262^{\mathrm{cA}}$ & $649^{\mathrm{bA}}$ & $1698^{\text {aA }}$ & $87^{\mathrm{aA}}$ & $89^{\mathrm{aA}}$ & $133 \mathrm{bA}^{\mathrm{b}}$ \\
\hline BUF & $197^{\text {a A }}$ & $170^{\text {а в }}$ & $175^{\text {а в }}$ & $73^{\text {a A }}$ & $73^{\mathrm{aA}}$ & 77 а в \\
\hline
\end{tabular}

Letras minúsculas diferentes na mesma linha e letras maiúsculas diferentes na mesma coluna, para os mesmos elementos, apresentam diferença significativa.

Tabela 3. Mediana da concentração hepática de cobre no final do experimento (dia 105) considerando o grupo controle (BUF) e os animais suplementados com cobre, separados em dois subgrupos, os que apresentaram intoxicação (BUFCu ICA) e os animais resistentes (BUFCu R).

\begin{tabular}{lccc}
\hline Grupos & $\mathrm{n}$ & Cu hepático (ppm) & Zn hepático (ppm) \\
\cline { 2 - 3 } & 2 & $2052^{\mathrm{a}}$ & $126^{\mathrm{a}}$ \\
BUFCu R & 4 & $1322^{\mathrm{b}}$ & $144^{\mathrm{a}}$ \\
BUF & 4 & $175^{\mathrm{c}}$ & $77^{\mathrm{b}}$ \\
\hline
\end{tabular}

Letras minúsculas diversas na coluna apresentam diferença significativa $(\mathrm{P}<0,0001)$. 
A tabela 4 apresenta os valores medianos do cobre sérico e da atividade da GGT nos grupos tratados com cobre e controle no decorrer do experimento. $\mathrm{O}$ grupo controle não apresentou nenhuma diferença para estas duas variáveis. No grupo BUFCu o cobre sérico apresentou grande variação de valores, ocasionada pela presença de animais intoxicados e resistentes. Nesse mesmo grupo, a atividade da GGT apresentou valores progressivos, com elevação da atividade enzimática nas últimas quinzenas em relação tanto às coletas anteriores quanto ao grupo controle.

Tabela 4. Medianas do cobre sérico e da atividade da GGT nas quinzenas do experimento nos grupos suplementados com cobre (BUFCu) e controle (BUF).

\begin{tabular}{ccccc}
\hline Quinzenas & \multicolumn{2}{c}{ Cobre sérico $(\mu \mathrm{Mol} / \mathrm{L})$} & \multicolumn{2}{c}{ GGT sérica $(\mathrm{U} / \mathrm{L})$} \\
\hline & BUFCu & BUF & BUFCu & BUF \\
\cline { 2 - 5 } 0 & 10,8 & 11,9 & $13^{\mathrm{B}}$ & 13 \\
1 & 11,4 & 14,3 & $16^{\mathrm{B}}$ & 12 \\
2 & 11,1 & 10,2 & $15^{\mathrm{B}}$ & 10 \\
3 & 10,5 & 11,9 & $16^{\mathrm{B}}$ & 12 \\
4 & 18,1 & 13,0 & $28^{\mathrm{B}}$ & 11 \\
5 & 17,2 & 10,8 & $28^{\mathrm{B}}$ & $12^{\mathrm{b}}$ \\
6 & 13,4 & 10,1 & $26^{\mathrm{aA}}$ & $9^{\mathrm{b}}$ \\
7 & 12,8 & 11,4 & 0,006 & 0,399 \\
\hline
\end{tabular}

Letras minúsculas diferentes na mesma linha e letras maiúsculas diferentes na mesma coluna para a mesma variável apresentam diferença significativa.

Observa-se que os valores de cobre sérico foram numericamente mais elevados na quarta e quinta quinzena, sendo está ultima o momento em que os dois bufalinos apresentaram quadro fatal de ICA. A eliminação destes dois animais dos valores medianos desta variável provocou a redução do cobre sérico observada nas últimas duas quinzenas.

Os valores da atividade da AST sérica durante o experimento se apresentaram inconstantes, com diferenças inclusive entre os animais do grupo controle. Não foram observadas diferenças nos valores da uréia sérica nos grupos BUFCu e BUF em nenhuma quinzena do experimento. Contudo, na subdivisão dos grupos na última quinzena, observaram-se valores superiores de uréia sérica para os animais intoxicados (BUF ICA) em relação aos resistentes (BUF R) e aos controles (BUF) (16,2; 5,3 e $5,0 \mathrm{mMol} / \mathrm{L}$ respectivamente). Em relação à creatinina sérica, os animais do grupo BUF ICA apresentaram valores maiores que os dos grupos BUF
R e BUF (228, 151 e $136 \mu \mathrm{Mol} / \mathrm{L}$ respectivamente), mas apenas um animal apresentou valores acima da normalidade (KANEKO; HARVEY; BRUSS, 1997).

\section{Discussão}

O modelo experimental empregado para intoxicação cúprica em ovinos, baseado num aumento gradativo de infusão de cobre a partir de uma dose fixa, segundo Machado (1998) e Soares (2004) se mostrou razoavelmente eficiente para induzir quadro de ICA em búfalos. Enquanto que no trabalho dos autores supracitados todos os ovinos desenvolveram o quadro clássico no prazo de 97 dias, em 105 dias um terço dos búfalos manifestou sintomas sugestivos da enfermidade. Deve-se lembrar inicialmente que os ovinos são classicamente conhecidos como a espécie mais susceptível à ICA, em especial, pelo fato desses 
animais apresentarem uma menor excreção biliar do cobre acumulado no fígado, decorrente de uma menor ligação entre o cobre hepático e a enzima responsável por esse mecanismo de excreção, a metalotioneína (HOWELL; GAWTHORNE, 1987; LÓPEZ-ALONSO et al., 2005).

Essa maior susceptibilidade pode ser avaliada pela quantidade de gramas de cobre / $\mathrm{kg}$ de peso metabólico ( $\mathrm{PV}{ }^{0,75}$ ) utilizada para provocar morte por ICA nas espécies de ruminantes. Enquanto que em ovinos, Soares (2004) utilizou em média 1,5 g cobre / kg PV ${ }^{0,75}$, no presente estudo necessitouse, em média de 4,1 mg de cobre / $\mathrm{kg} \mathrm{PV}{ }^{0,75}$ para provocar ICA em búfalos.

Constituiu-se uma surpresa o surgimento de um quadro sintomatológico atípico, que não o clássico. A ausência de sintomas clássicos como a hemoglobinúria e icterícia precedendo a morte gerou inicialmente perplexidade quanto ao diagnóstico. Pensava-se que pudesse ser um quadro hemorrágico abomasal ou intestinal, mas na necropsia isto não foi constatado. Posteriormente, detectaram-se altas quantidades de cobre no fígado dos mesmos, semelhante à observada no quadro clássico de ICA e superior ao constatado nos búfalos resistentes, passando-se a admitir um efeito prejudicial direto do excesso de cobre desencadeando o quadro atípico.

É digno de nota que numa descrição de surto de ICA em vacas bovinas leiteiras Bradley (1993) apenas cita que uma das seis fêmeas acometidas apresentou anorexia, conteúdo ruminal ressecado, ausência de hemoglobinúria, mucosas amareladas e coloração enegrecida nos rins, mas altos teores de cobre no fígado e rins. Mesmo assim, o autor atribuiu a morte pela ICA; já em búfalos, tal quadro nunca foi relatado.

Outro fator relevante ao diagnóstico da ICA em búfalos é a intensa desidratação apresentada pelos animais, o que dificulta a observação da hemoglobinúria, onde no animal com quadro clínico, no segundo dia da manifestação da doença, foi necessária intensa e prolongada estimulação para que se conseguisse obter uma amostra de urina. Tal fator pode contribuir para a falha do diagnóstico a campo de ICA em búfalos.

Em relação ao peso vivo deve ser ressaltada a grande capacidade dos animais resistentes continuarem a apresentar ganho de peso em comparação com os que desenvolveram ICA, quando estes a partir das últimas quinzenas tiveram franca perda de peso vivo.

Merecem reflexão os mecanismos desencadeados pelos animais resistentes à ICA. Segundo dados da última biópsia, os búfalos resistentes acumularam no fígado 1,55 vezes menos cobre que os animais que sucumbiram de ICA. Embora o menor acúmulo de cobre fosse explícito nos resistentes, não houve elevação nos teores hepáticos de zinco, o que poderia promover maior resistência, por estímulo de produção de metalotioneínas nesse órgão. Um outro mecanismo de proteção contra o acúmulo exagerado de cobre no organismo seria por diminuição na sua disponibilidade, por meio de menor absorção intestinal (UNDERWOOD; SUTTLE, 1999).

Com relação aos valores elevados de AST encontrados, em estudo com búfalos da raça Murrah, Yang et al. (1998) encontraram valores similares aos constatados no grupo controle do presente trabalho, ratificando os resultados obtidos.

As areas de necrose centrolobular observadas durante esse experimento já foram descritas na intoxicação crônica por cobre (KELLY, 1993; CULLEN, 2007), e estão associadas à hipoxia. Enquanto as áreas de hiperplasia periportal e degeneração gordurosa podem estar relacionadas ao início da fase de regeneração hepática característica dessa intoxicação (CULLEN, 2007).

\section{Conclusões}

Os bufalinos, em condições experimentais, podem ser acometidos por dois quadros sintomatológicos distintos de ICA, o clássico e outro atípico caracterizado por longo período 
de hiporexia, emagrecimento destacado, apatia intensa, desidratação e ausência de hemoglobinúria macroscópica.

Parte dos búfalos se mostrou resistente à ICA a despeito da administração de altas quantidades cobre. Em búfalos que apresentem quadros clínicos com anorexia, perda de peso, apatia, desidratação, oligúria e morte, a intoxicação cúprica acumulativa pode ser considerada como um possível diagnóstico mesmo sem a observação macroscópica de hemoglobinúria.

\section{Agradecimentos}

Os autores agradecem ao CNPq e a FAPESP pela concessão de bolsas.

Pesquisa financiada pela FAPESP: processo número 2005/03204-0.

\section{Referências}

AUZA, N. J.; OLSON, W. G.; MURPHY, M. J.; LINN, J. G. Diagnosis and treatment of copper toxicosis in ruminants. Journal of American Veterinary Medical Association, Schaumburg, v. 214, n. 11, p. 1624-1628, 1999.

BIDEWELL, C.; LIVESEY, C. Copper poisoning: an emerging disease in dairy cattle. State Veterinary Journal, London, v. 12, n. 2, p. 16-19, 2002.

BRADLEY, C. H. Copper poisoning in a dairy herd fed a mineral supplement. Canadian Veterinary Journal, Ottawa, v. 34, n. 5, p. 287-292, 1993.

CARDOSO, E. C.; MCDOWELL, L. R.; VALE, W. G.; VEIGA, J. B.; SIMÃO NETO, M.; WILKINSON, N. S.; LOURENÇO JUNIOR, J. B. Copper and molybdenum status of cattle and buffaloes in Marajó Island, Brazil. International Journal of Animal Science, Sonipat, v. 12, n. 1, p. 57-60, 1997.

CULLEN, J. M. Liver, biliary system, and exocrine pancreas. In: McGAVIN, M. D.; ZACHARY, J. F. Pathological basis of veterinary disease. $4^{\text {th }}$ ed. Saint Louis: Elsevier, 2007. p. 393-461.

HOOF, B.; BOERMANS, H. J.; BAIRD, J. D. Retrospective study of toxic metal analyses request at a veterinary diagnostic toxicology laboratory in Ontario (1990-1995). Canadian Veterinary Journal, Ottawa, v. 39, n. 1, p. 39-43, 1998.

HOWELl, J. M.; GAWTHORNE, J. M. Copper in animals and man. Boca Raton: CRC Press, 1987.

KANEKO, J. J.; HARVEY, J. W.; BRUSS, M. L. Clinical biochemistry of domestic animals. $5^{\text {th }}$ ed. San Diego: Academic Press, 1997.

KELLY, W. R. The liver and biliary systems. In: JUBB, K. V. F; KENNEDY, P. C.; PALMER, N. Pathology of domestic animals. $4^{\text {th }}$ ed. San Diego: Academic Press, 1993. v. 2. p. 319-406.

LÓPEZ-ALONSO, M; PRIETO, F.; MIRANDA, M.; CASTILLO, C.; HERNANDÉZ, J.; BENEDITO, J. L. The role of metallothionein and zinc in hepatic copper accumulation in cattle. Veterinary Journal, Amsterdam, v. 169, n. 2, p. 262-267, 2005.

MACEDO, M. P.; WECHSLER, F. S.; RAMOS, A. A.; AMARAL, J. B.; SOUZA, J. C.; RESENDE, F. D.; OLIVEIRA, J. D. Composição físico-química e produção do leite de búfalas da raça Mediterrâneo no oeste do Estado de São Paulo. Revista Brasileira de Zootecnia, Viçosa, v. 30, n. 3, Suppl. 1, p. 1084-1088, 2001.

MACHADO, C. H. Uso do tetratiomolibdato no tratamento de intoxicação cúprica experimental em ovinos: avaliações clínica e toxicológica. 1998. Tese. (Doutorado em Clínica Veterinária) - Faculdade de Medicina Veterinária e Zootecnia da Universidade de São Paulo, São Paulo.

MINITAB. The student edition of MINITAB Statistical software adapted for education. Release 13.0. User's Manual. New York: Addison-Wesley, 2000. CD-ROM.

ORTOLANI, E. L. Efeitos da suplementação dietética de molibdênio e enxofre sobre a infestação de Haemonchus contortos (Rudolphi, 1803), em ovinos. Estudo de alguns aspectos do metabolismo de cobre, sódio e da resposta celular do hospedeiro. 1997. Tese (Livre-Docência) - Faculdade de Medicina Veterinária e Zootecnia da Universidade de São Paulo, São Paulo.

. Intoxicação cúprica acumulativa em ovinos. In: CONGRESSO BRASILEIRO DE BUIATRIA, 5., 2003, Salvador. Anais... Salvador: Associação Brasileira de Buiatria, 2003. p. 113-114.

SOARES, P. C. Efeitos da intoxicação cúprica e do tratamento com Tetratiomolibdato sobre a função renal e o metabolismo oxidativo de ovinos. 2004. Tese. (Doutorado em Ciência Veterinária) - Faculdade de Medicina Veterinária e Zootecnia da Universidade de São Paulo, São Paulo. 
UNDERWOOD, E. J.; SUTTLE, N. F. The mineral nutrition of livestock. $3^{\text {th }}$ ed. Wallingford: CABI Publishing, 1999.

YANG, Q.; MAO, W.H.; FERRE, I.; BAYÓN, J.E.; MAO, X. Z.; GONZÁLEZ-GALLEGO, J. Plasma aspartate aminotransferase (AST), glutamate dehydrogenase (GLDH) and gamma-glutamyl transpeptidase (GGT) activities in water buffaloes with experimental subclinical fasciolosis. Veterinary Parasitology, Amsterdam, v. 78, n. 2, p. 129-136, 1998.

ZICARELLI, L.; MACRI, A.; VITTORIA, A.; PADULA, P.; COSTANTINI, S.; RANIA, V.; GIORDANO, R. Intossicazione da rame in vitelli bufalini. Rivista di Zootecnia i Veterinaria, Milan, v. 9, n. 4, p. 246-251, 1981. 
